\title{
Genomic profiling of synchronous triple primary tumors of the lung, thyroid and kidney in a young female patient: A case report
}

\author{
LING PENG $^{1 *}$, ZHU ZENG $^{1 *}$, XIAODONG TENG ${ }^{2}$, ZHEN CHEN $^{2}$, LILI LIN $^{3}$, HUA BAO $^{4}$, \\ YANG W. SHAO ${ }^{4}$, YINA WANG ${ }^{1}$, YONGQUAN DONG ${ }^{5}$ and QIONG ZHAO $^{1}$ \\ Departments of ${ }^{1}$ Thoracic Oncology, ${ }^{2}$ Pathology and ${ }^{3} \mathrm{PET} / \mathrm{CT}$, The First Affiliated Hospital, School of Medicine, \\ Zhejiang University, Hangzhou, Zhejiang 310003, P.R. China; ${ }^{4}$ Translational Medicine Research Institute, \\ Geenseeq Technology Inc., Toronto, ON M5G1L7, Canada; ${ }^{5}$ Department of Respiratory Disease, \\ YinZhou Second Hospital, Ningbo, Zhejiang 315040, P.R. China
}

Received July 16, 2017; Accepted August 1, 2018

DOI: $10.3892 / \mathrm{ol} .2018 .9334$

\begin{abstract}
Synchronous multiple primary malignant tumors (MPMTs) are rare in young adults. Genomic profiling of synchronous MPMTs has not been systematically investigated to elucidate their genetic associations, but may be important to assist diagnosis and guide appropriate treatment strategy. In the present study, mutation profiling was performed using targeted next generation sequencing (NGS) on 416 cancer-related genes in synchronous triple primary tumors of the lung, kidney and thyroid in a 32-year-old female patient. The patient was diagnosed with moderately differentiated lung adenocarcinoma (T2aNOM0; stage IB), renal clear cell carcinoma (T1aNOM0; stage I), and thyroid papillary carcinoma (T1NlaM0, stage III) by pathological assessments. Clinically actionable mutations in EGFR and BRAF genes were identified in the lung and the thyroid lesions, respectively. Three tumors demonstrated distinct genomic profiles, suggesting that all tumors were independent primary tumors, which was consistent with histopathological assessment. Three potential germline cancer susceptibility mutations were shared between this patient and her father who was diagnosed with lung cancer. The present results demonstrated that, in the context of identical germline background and environmental exposure, multiple synchronous tumors in the same patient may have distinct mutation profiles and can be driven by distinct molecular
\end{abstract}

Correspondence to: Professor Qiong Zhao, Department of Thoracic Oncology, The First Affiliated Hospital, School of Medicine, Zhejiang University, 79 Qingchun Road, Hangzhou, Zhejiang 310003, P.R. China

E-mail: doczq.2008@hotmail.com

*Contributed equally

Key words: synchronous carcinoma, multiple primary malignant tumors, next generation sequencing, mutation profiling, inherited cancer susceptibility events. Combination therapies may need to be considered during treatment decision-making.

\section{Introduction}

Patients with multiple primary malignant tumors (MPMTs) are often described as synchronous or metachronous tumors based on their chronology of presentation (1). Synchronous MPMTs are defined as that additional primary tumors are diagnosed within 6 months of the primary cancer, which are relatively rare, especially in young adults. The confirmation of synchronous MPMTs puts forward important diagnostic and therapeutic challenges. Genomic profiling of synchronous MPMTs have not been systematically investigated, but may be important to reveal the tumorigenesis mechanism, assist diagnosis and guide treatment decision-making.

Here, we reported a 32-year-old female patient, who has a family history of lung cancer found in her father, and was diagnosed with synchronous lung adenocarcinoma, thyroid carcinoma, and renal carcinoma by comprehensive histopathologic evaluation. Targeted next generation sequencing (NGS) was used to characterize the genomic profiling and identify genetic relationships among MPMTs within this patient. Moreover, we identified potential germline cancer susceptibility mutations shared between this patient and her father, which could possibly explain the early onset of cancers.

\section{Patients and methods}

Patient information and sample collection. The patient was informed and written consent was collected according to the ethic regulation of The First Affiliated Hospital of Zhejiang University (Hangzhou, China). The tests were performed in a centralized clinical testing center (Nanjing Geneseeq Technology Inc., Nanjing, China). All methods were performed in accordance with the relevant guidelines and regulations in The First Affiliated Hospital of Zhejiang University.

Targeted NGS. Histological slides from FFPE tissue specimens were reviewed for tumor content and the tumor area was marked by a pathologist. Five of $10 \mu \mathrm{m}$ tissue sections from 
tumor FFPE samples were used for genomic DNA extraction with QIAamp DNA FFPE Tissue kit (Qiagen Inc., Toronto, ON, Canada) following the manufacturer's instructions. Genomic DNA from whole blood sample was extracted using DNeasy blood and tissue kit (Qiagen Inc.) according to manufacturer's protocol. Sequencing libraries were prepared using the KAPA Hyper Prep kit (KAPA Biosystems Inc., Wilmington, MA, USA) with recommended standard protocol. Hybridization capture was carried out following the methods as previously described (2). The target-enriched library was then sequenced on HiSeq4000 NGS platforms (Illumina, San Diego, CA, USA). Sequencing depth was $\sim 600 \mathrm{X}$ for tumor samples and $\sim 100 \mathrm{X}$ for normal blood control samples.

Sequencing data analysis. Detailed data analysis methods were discussed in Shu et al (2). Briefly, sequencing reads were mapped to the reference sequence hg19 (Human Genome version 19) using Burrows-Wheeler Aligner (BWA-mem, version 0.7.12) (3). Local realignment around indels and base quality score recalibration were applied with the Genome Analysis Toolkit (version 3.4.0) (4). VarScan (version 2.3.7) was employed for detection of somatic mutations with default parameters (5). Annotation was performed using ANNOVAR (version 2017Jun01) with default parameters (6). Genome-wide copy number profiling was analyzed by FACETS (version 0.5.6) software with default parameters (7).

In order to identify the relationships among the triple primary carcinomas, pairwise spearman correlation of the copy number variations (CNVs) between the three tumors was calculated, and compared with that of CNVs derived from 10 unrelated patients with single primary tumors.

We screened the possible germline cancer susceptibility mutations using the following criteria: i) Shared germline mutations between the patient and her father; ii) missense or nonsense mutations; iii) frequency $<1 \%$ in 1,000 healthy people genomics (8) and 65,000 healthy people exomes (9); iv) at least one of the algorithms (SIFT, Polphen2, and MutationTaster, calculated by ANNOVAR) predicted the amino acid changes to be damaging mutations.

\section{Results}

Clinical history. A 32-year-old female patient was referred to The First Affiliated Hospital of Zhejiang University in November 2015 due to a suspected nodule in the left lower lobe of the lung (Fig. 1A) detected by routine physical exam in May 2015. The patient had no smoking history and experienced no symptoms related to her malignancies. Pre-operational examinations identified another nodule in the right lobe of thyroid (Fig. 1B) and a mass in the left upper kidney (Fig. 1C). Positron emission tomography-computed tomography (PET/CT) scan further confirmed the lung nodule with elevated standardized uptake values (SUVs) (Fig. 1D), and showed a focal fludeoxyglucose (FDG)-avid nodule with calcification in the right upper thyroid lobe, and a $3 \times 2 \mathrm{~cm}$ mass in the left kidney (Fig. 1E and F).

Considering her synchronous triple tumors, the surgeons in our multidisciplinary team decided that consecutive resections need to be performed. Partial nephrectomy of the left kidney was first performed in December 2015. After
6 months, she received right thyroidectomy with modified radical neck dissection. One month later, she received right upper lobectomy of the lung and radical lymphadenectomy. Following all the surgical resections, the patient received 4 cycles of adjuvant chemotherapy with pemetrexed/cisplatin. Histopathological evaluation of the synchronous triple carcinomas revealed distinct tumor morphologies (Fig. 2). The tumor in kidney was identified as renal clear cell carcinoma with papillary structure (pT1aN0M0, stage I). Immunohistochemical (IHC) staining were positive for KSP-cadherin, VHL, PAX8, CK(pan), Vimentin, CK7, P504S, and no expression of IXTFE3, CD10, E-cadherin, CD117, CD15, HMB45, and Melan-A were detected. The tumor in thyroid was diagnosed as thyroid papillary carcinoma (pT1N1aM0, stage III) with right central compartment lymph node metastasis, but without right recurrent laryngeal nerve lymph node metastasis. Post-operative diagnosis of the tumor in lung was moderately differentiated lung adenocarcinoma with positive IHC staining for TTF-1, Napsin A and CD34, whereas no expression of p63, D2-40, PAX8, and TG were detected. The tumor invaded into the visceral pleura, with pathological staging of pT2aN0M0, stage IB.

Of note, the patient's father (58-year-old) visited our hospital in July 2015 due to shingles. However, blood test revealed elevated serum levels of carcinoembryonic antigen (CEA) and CA-125. A suspected lung nodule was detected by $\mathrm{CT}$ scan, and the mediastinal lymph node (4R) was diagnosed as poorly differentiated adenocarcinoma of the lung (cT1aN2M0, stage IIIA) by endobronchial ultrasound with transbronchial needle aspiration (Fig. 3). Lung surgery was performed after 2 cycles of neoadjuvant chemotherapy with pemetrexed/cisplatin. The father has a 20 pack-year smoking history.

Mutation profiling revealed independency of the triple primary malignancies. To determine the genomic heterogeneity and assess the genetic relationships among the three tumors within this patient, we performed targeted NGS of 416 cancer-related genes. In total, 7 missense mutations were identified in 3 tumors (Table I). Somatic mutation of $B R A F$ p.V600E (COSMIC476) and MAP3K1 p.A109V were identified in the thyroid tumor with variant allele frequency (VAF) of 12.96 and $1 \%$, respectively. The lung tumor harbored somatic mutations EGFR p.G719S (COSM6252), EGFR p.E709A (COSM13427), TP53 p.Y205C (COSM43947) and CDK12 p.G28D with VAF of $58.92,61.69,58.07$ and $1.88 \%$, respectively. ROS1 p.R2072Q (COSM3157658) was the only somatic mutation identified in the renal tumor with VAF of $1.06 \%$. Clearly, the thyroid and lung tumor were driven by $B R A F$ and $E G F R$ oncogenic mutations, respectively. There were no shared somatic mutations among these three tumors, indicating these tumors were of independent origins. However, the lung tumor from her father harbored distinct somatic mutations of TP53 p.R273H (COSM 10660) and RB1 p.Q384fs (COSM3724512) without other well-known oncogenic mutations.

To further test the origins of MTMPs, we characterized the copy number profiles of the three tumors. Similar to the patterns of point mutations discussed above, the copy number profiles of these three tumors were very different (Fig. 4A), 


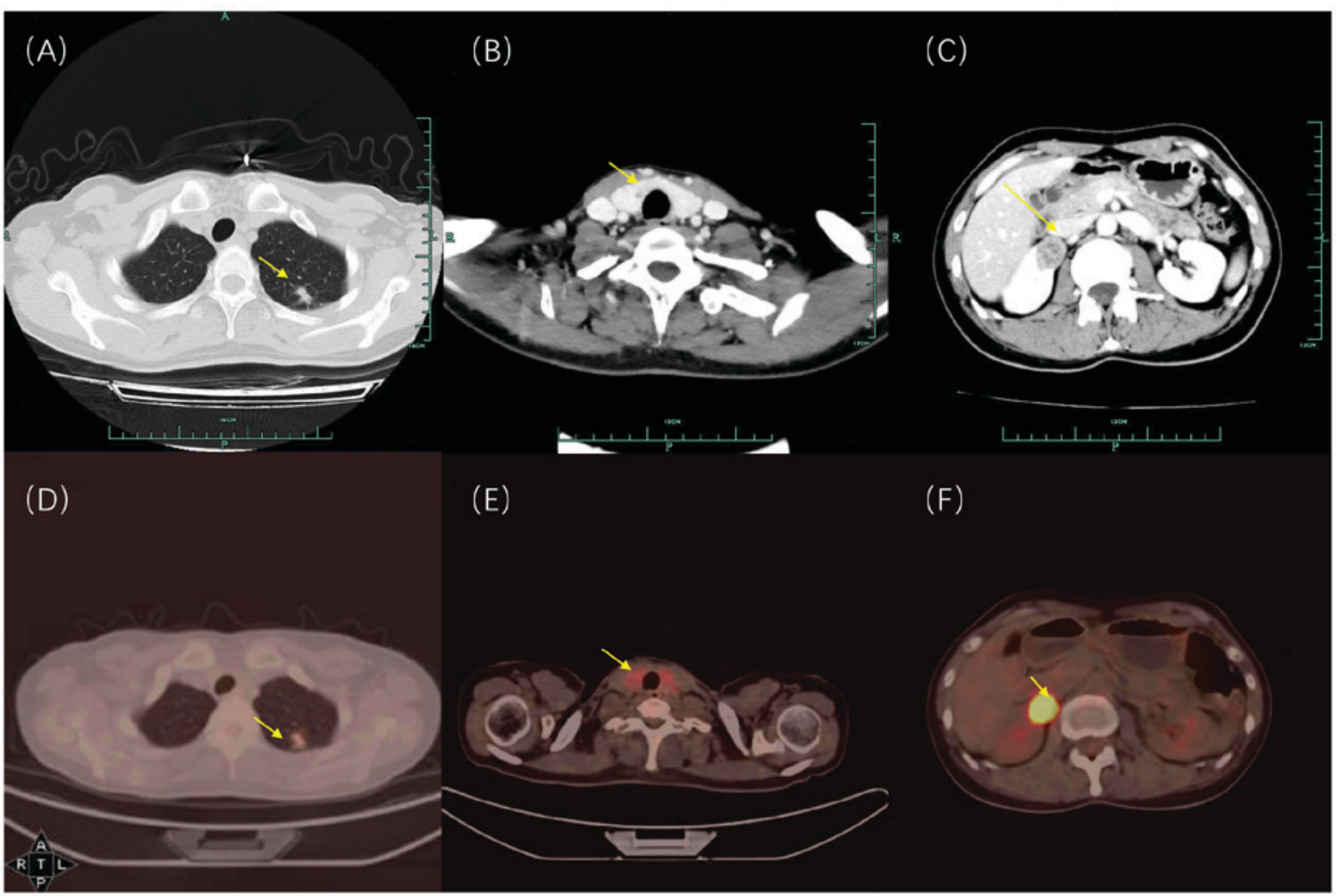

Figure 1. CTs and PET/CTs of tumor lesions at diagnosis. (A-C) Representative images of CT scan. (D-F) Representative images of PET-CT scan. The arrows indicate lesions of interest. (A, D) Lung adenocarcinoma located in the left upper lobe of the lung; (B and E) thyroid papillary carcinoma in the right thyroid; (C and F) renal clear cell carcinoma in the kidney. PET, positron emission tomography; CT, computed tomography.

(A) Thyroid cancer

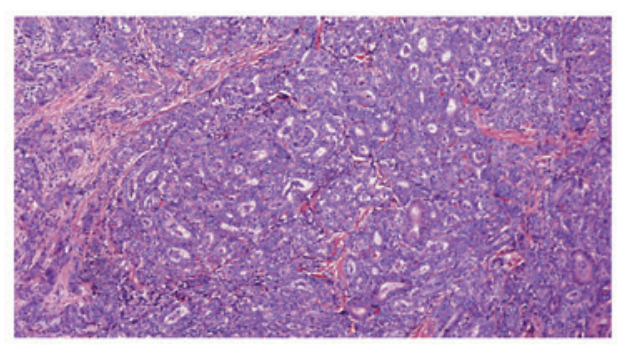

(B) Renal cancer

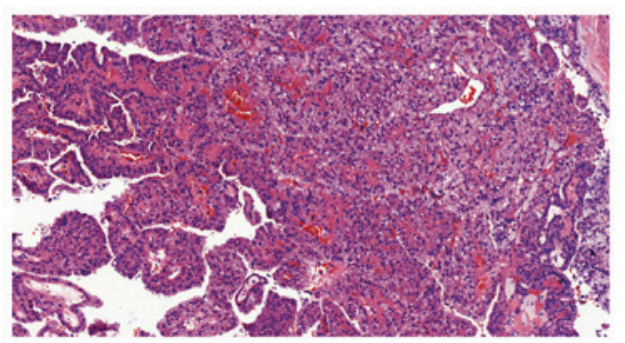

(C) Lung cancer

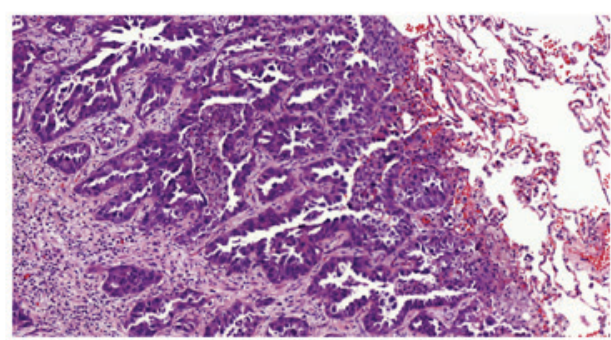

Figure 2. Locations of the synchronous triple primary tumors and H\&E staining. Image courtesy of Professor Konstantin J Dedes from University of Zurich. (A) Thyroid papillary carcinoma. (B) Renal clear cell carcinoma. (C) Lung adenocarcinoma. H\&E staining; original magnification, x20. H\&E, hematoxylin and erosin staining.

consistent with the independent nature of these tumors. Next, we assessed the degree of similarity of copy number alterations (CNAs) among these three tumors within the patient and compared to the similarity among randomly 
Table I. Somatic mutations of the synchronous triple carcinomas.

\begin{tabular}{llccc}
\hline Gene & \multicolumn{1}{c}{ Genetic alterations } & Lung cancer (\%) & Thyroid cancer (\%) & Renal cancer (\%) \\
\hline BRAF & p.V600E (c.T1799A) & - & 12.96 & - \\
$C D K 12$ & p.G28D (c.G83A) & 1.88 & - & - \\
$E G F R$ & p.G719S (c.G2155A) & 58.92 & - & - \\
$E G F R$ & p.E709A (c.A2126C) & 61.69 & - & - \\
$M A P 3 K 1$ & p.A109V (c.C326T) & - & 1 & 1.06 \\
ROS1 & p.R2072Q (c.G6215A) & - & - & - \\
$T P 53$ & p.Y205C (c.A614G) & 58.07 & - & \\
\hline
\end{tabular}

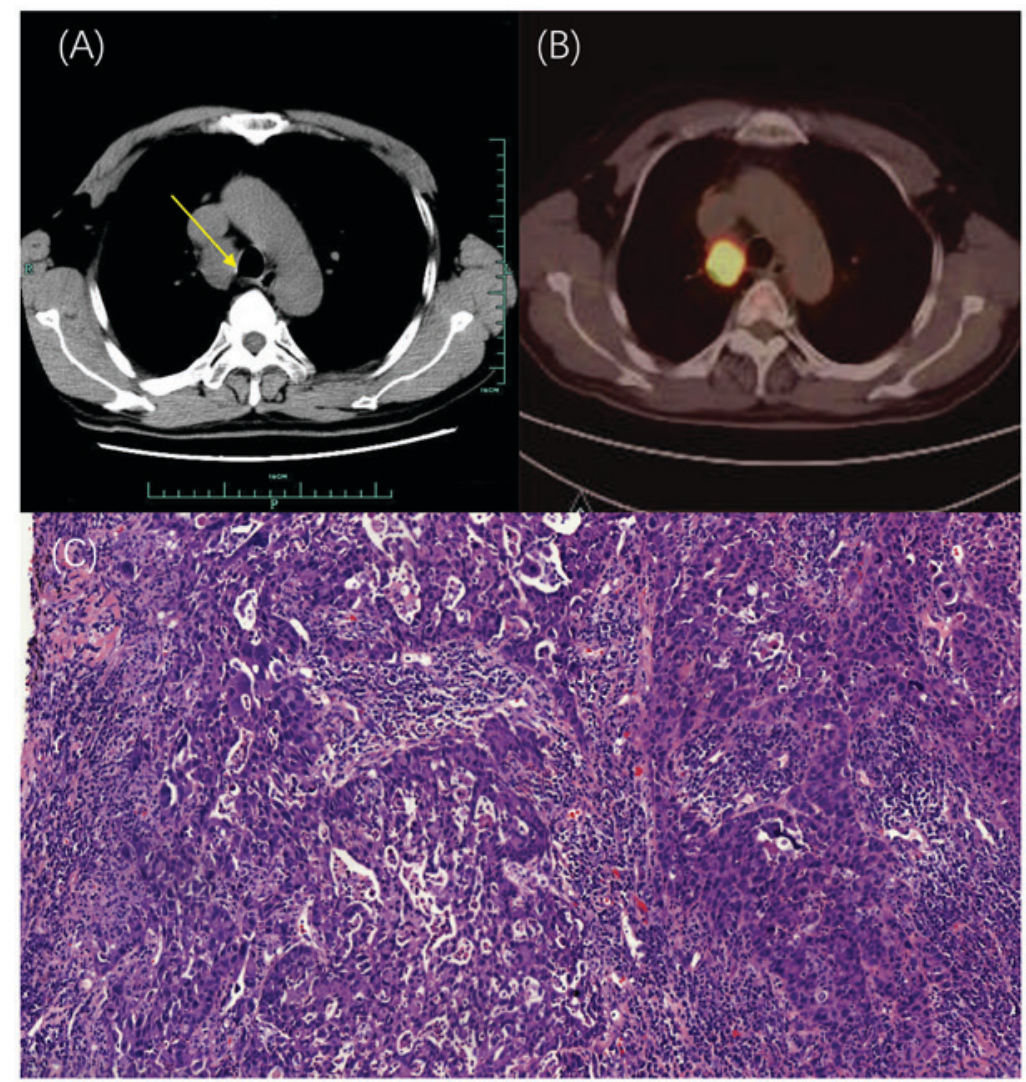

Figure 3. Pulmonary CT-scan and H\&E staining of lung tumor from the father. The arrows indicate lesions of interest. (A) CT-scan picture showing the mediastinal lymph node; (B) PET/CT picture of the lesions of interest. (C) Lung adenocarcinoma after surgery. Hematoxylin and erosin staining; original magnification, x20. H\&E, hematoxylin and erosin staining; PET, positron emission tomography; CT, computed tomography.

selected patients with only single primary tumors. If the independent origin was true, three tumors from this patient should be no more similar to each other than tumors from different patients. Indeed, pairwise spearman correlations of CNAs among three tumors were not significantly different with the background distributions based on pairwise correlations among 10 unrelated patients $(\mathrm{P}=0.93$, Wilcox rank-sum test) (Fig. 4B). Taken together, results from both point mutations and CNAs indicated that the MTMPs in this patient are independent primary tumors.

Since the father of this patient was also diagnosed with lung cancer harboring distinct somatic mutations, we sought to identify potential germline cancer susceptibility mutations shared between the patient and her father. We screened the possible damaging germline mutations using the following criteria: i) Functional (missense or nonsense); ii) rare $(<1 \%)$ or not exist in 1,000 genomes and 650,000 exomes database; iii) predicted damaging functions of amino acid changes. Three potential germline cancer susceptibility mutations (GATA6 p.S184N, BRD4 p.P1170L, and QKI p.T245A) were identified. These inherited mutations may be associated with the accumulation of somatic aberrations, which promote cancer development in this patient.

\section{Discussion}

The incidence of MPMTs has been reported to vary from 0.7 to $11.7 \%$ (10), which has increased owing to prolonged survival of cancer patients and advances in diagnostic techniques (1). Synchronous tumors are defined as additional 

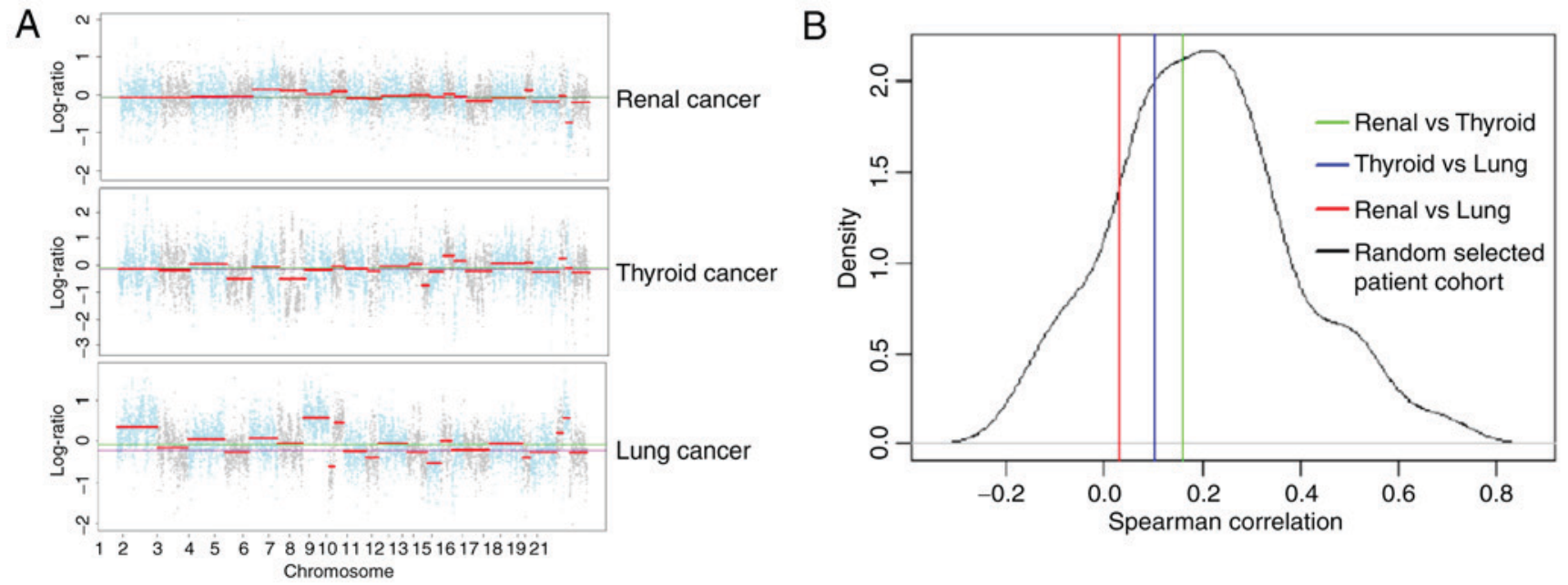

Figure 4. Genome-wide copy number profiles in synchronous triple tumors. (A) Top, middle and bottom panel represent log2 normalized depth ratio between tumor and normal control for renal, thyroid and lung tumor, respectively. Chromosomes were alternating in blue and gray colors. (B) Pairwise spearman correlations of gene-level CNAs among three tumors were compared with the background distributions based on pairwise correlations among 10 randomly selected unrelated patients with single tumors. Correlation of CNAs between renal and thyroid tumor, thyroid and lung, renal and lung are 0.18 (green line), 0.11 (blue line) and 0.02 (red line), respectively. A total of 90 pairwise correlations among 10 unrelated patients were plotted as background distribution (black curve, density plot). CNAs, copy number alterations.

primary tumors occurring within 6 months of diagnosis of the first primary tumor (1). However, such cases require strict exclusion of possible metastasis to either site and to confirm the primary nature of each malignancy for better outcome of management and survival benefit. This study investigated histopathological and genomic profiling of the synchronous triple tumors within a young female patient. Histopathological evaluation of the synchronous MPMTs revealed the distinct tumor morphology. In addition, the mutation profiling demonstrates that the development of these three synchronous primary tumors were clonally independent. To our knowledge, there have been no similar reported cases of synchronous triple primary tumors occurring in lung, thyroid and kidney. In addition, studies have shown that half of all clinical T1 lesions can be detected by FDG PET-CT, which suggests that FDG PET-CT is not only useful to identify distant metastases of the primary tumor, but also for the purpose of detecting additional primary synchronous cancers (11).

Our results further indicate that the multiple synchronous tumors in the same patient can be driven by distinct oncogenic events. Thus, combination of targeted therapies may need to be considered during treatment decision-making. EGFR signaling pathway is one of the most frequently altered pathways in lung adenocarcinoma (12). The G719S mutation identified in the lung tumor occurs with a frequency of $2-3 \%$ in EGFR-mutated lung cancers (13). This mutation is associated with increased sensitivity to the EGFR TKIs including erlotinib and gefitinib. The BRAF V600E mutation identified in the thyroid carcinoma of this patient is a well-known driver mutation (14), which is also a prognostic factor for thyroid cancer (15), and can be targeted by inhibitors of mutated BRAF.

Multiple primary synchronous malignancies may be due to shared genetic mutations if any common carcinogenic factor cannot be found $(16,17)$. The early onset of the malignant disease in this 32-year-old female patient suggests possible germline mutations in cancer susceptibility genes that accelerated the accumulation of somatic aberrations promoting cancer development. Although no shared germline mutations were identified in well-known cancer susceptibility genes such as MMR-related genes, BRCA1/2, $T P 53, R B 1$ and $A P C$, we identified three unreported germline mutations, which could possibly explain the early onset cancers in this young patient. However, other hereditary factors might still exist.

In summary, we reported a case of a 32-year-old female patient with synchronous triple primary carcinomas with independent clonal origins. To our knowledge, this is the first report of synchronous lung, thyroid and renal cell cancer occuring in one patient. Our results demonstrate that in the context of identical genetic background and environmental exposure history, multiple synchronous tumors in the same patient can have distinct mutational profiles and can be driven by distinct molecular events.

\section{Acknowledgements}

The present study was supported by Natural Science Foundation of China (grant no 81402179 to Dr Ling Peng). We thank Professor Ying-Jie Wang from The First Affiliated Hospital, Zhejiang University for providing helpful insights and suggestions. We thank Professor Konstantin Dedes from University of Zurich for helping us to prepare image of Fig. 1.

\section{Conflicts of interest}

Hua Bao and Yang W. Shao are the shareholders or employees of Geneseeq Technology Inc.

\section{References}

1. Testori A, Cioffi U, De Simone M, Bini F, Vaghi A, Lemos AA, Ciulla MM and Alloisio M: Multiple primary synchronous malignant tumors. BMC Res Notes 8: 730, 2015. 
2. Shu Y, Wu X, Tong X, Wang X, Chang Z, Mao Y, Chen X, Sun J, Wang Z, Hong Z, et al: Circulating tumor DNA mutation profiling by targeted next generation sequencing provides guidance for personalized treatments in multiple cancer types. Sci Rep 7: 583, 2017.

3. Li H and Durbin R: Fast and accurate short read alignment with Burrows-Wheeler transform. Bioinformatics 25: 1754-1760, 2009.

4. McKenna A, Hanna M, Banks E, Sivachenko A, Cibulskis K, Kernytsky A, Garimella K, Altshuler D, Gabriel S, Daly M and DePristo MA: The Genome Analysis Toolkit: A MapReduce framework for analyzing next-generation DNA sequencing data. Genome Res 20: 1297-1303, 2010.

5. Koboldt DC, Zhang Q, Larson DE, Shen D, McLellan MD, Lin L, Miller CA, Mardis ER, Ding L and Wilson RK: VarScan 2: Somatic mutation and copy number alteration discovery in cancer by exome sequencing. Genome Res 22: 568-576, 2012.

6. Wang K, Li M and Hakonarson H: ANNOVAR: Functional annotation of genetic variants from high-throughput sequencing data. Nucleic Acids Res 38: e164, 2010.

7. Shen R and Seshan VE: FACETS: Allele-specific copy number and clonal heterogeneity analysis tool for high-throughput DNA sequencing. Nucleic Acids Res 44: e131, 2016.

8. 1000 Genomes Project Consortium, Auton A, Brooks LD, Durbin RM, Garrison EP, Kang HM, Korbel JO, Marchini JL, McCarthy S, McVean GA and Abecasis GR: A global reference for human genetic variation. Nature 526: 68-74, 2015.

9. Lek M, Karczewski KJ, Minikel EV, Samocha KE, Banks E, Fennell T, O'Donnell-Luria AH, Ware JS, Hill AJ, Cummings $\mathrm{BB}$, et al: Analysis of protein-coding genetic variation in 60,706 humans. Nature 536: 285-291, 2016.
10. Demandante CG, Troyer DA and Miles TP: Multiple primary malignant neoplasms: Case report and a comprehensive review of the literature. Am J Clin Oncol 26: 79-83, 2003.

11. Little SG, Rice TW, Bybel B, Mason DP, Murthy SC, Falk GW, Rybicki LA and Blackstone EH: Is FDG-PET indicated for superficial esophageal cancer? Eur J Cardiothorac Surg 31: 791-796, 2007.

12. Ding L, Getz G, Wheeler DA, Mardis ER, McLellan MD, Cibulskis K, Sougnez C, Greulich H, Muzny DM, Morgan MB, et al: Somatic mutations affect key pathways in lung adenocarcinoma. Nature 455: 1069-1075, 2008.

13. Mitsudomi T and Yatabe Y: Epidermal growth factor receptor in relation to tumor development: EGFR gene and cancer. FEBS J 277: 301-308, 2010.

14. Yarchoan M, LiVolsi VA and Brose MS: BRAF mutation and thyroid cancer recurrence. J Clin Oncol 33: 7-8, 2015.

15. Li C, Lee KC, Schneider EB and Zeiger MA: BRAF V600E mutation and its association with clinicopathological features of papillary thyroid cancer: A meta-analysis. J Clin Endocrinol Metab 97: 4559-4570, 2012.

16. Di Silverio F, Sciarra A, Flammia GP, Mariani M and De Vico A: Multiple primary tumors: 17 cases of renal-cell carcinoma associated with primary tumors involving different steroid-hormone target tissues. World J Urol 15: 203-209, 1997.

17. Hayat MJ, Howlader N, Reichman ME and Edwards BK: Cancer statistics, trends, and multiple primary cancer analyses from the Surveillance, Epidemiology, and End Results (SEER) Program. Oncologist 12: 20-37, 2007. 